\title{
Examples of practical activities related to public understanding of radiation risk following the Fukushima nuclear accident
}

\author{
Wataru Naito $^{1, a,{ }^{*}}$, Motoki Uesaka ${ }^{1}$, Yujiro Kuroda ${ }^{2,3}$, Takahiko Kono ${ }^{4}$, Akihiro Sakoda ${ }^{5}$ \\ and Hiroko Yoshida ${ }^{6}$ \\ ${ }^{1}$ Research Institute of Science for Safety and Sustainability, National Institute of Advanced Industrial Science and Technology (AIST), \\ Tsukuba, Ibaraki-Pref., Japan. \\ ${ }^{2}$ Fukushima Medical University, Fukushima, Fukushima-Pref., Japan. \\ ${ }^{3}$ Fukushima Prefectural Centre for Environmental Creation, Miharu, Fukushima-Pref., Japan. \\ ${ }^{4}$ Sector of Fukushima Research and Development, Japan Atomic Energy Agency, Iwaki, Fukushima-Pref., Japan. \\ ${ }^{5}$ Ningyo-toge Environmental Engineering Center, Japan Atomic Energy Agency, Kagamino, Okayama-Pref., Japan. \\ ${ }^{6}$ Radioisotope Research and Education Center, Tohoku University, Sendai, Miyagi-Pref., Japan.
}

Received: 23 November 2020 / Accepted: 24 November 2020

\begin{abstract}
After the Fukushima nuclear accident in Japan, a number of practical activities related to public understanding (PU) of radiation risks were implemented inside and outside Fukushima Prefecture. The various noteworthy approaches and strategies behind those practical activities have not been organized and made explicit thus far. In this study, we have organized the noteworthy practical activities related to PU of radiation risks following the Fukushima nuclear accident, and discussed them mainly from the standpoints of communication strategies and approaches. As several examples demonstrate, efforts to contextualize and localize radiation risk in various forms were observed during post-accident recovery in Fukushima, and these efforts were confirmed, through actual experiences, to be an important component of effective PU activities of radiation risks. Community-based or citizen science approaches, such as having affected residents or citizens to measure radioactivity, have contributed to the PU of radiological situations, but some challenges, such as ethical aspects and the handling of uncertainty, have also been revealed. In the era of information and communications technology, a number of citizens, experts, and agencies have made social media a popular platform for disseminating radiation risk messages to the public and have demonstrated that social media can play an important role in providing radiological risk information. The knowledge and lessons learned from the practical activities discussed in this study can be useful in enhancing PU of risks not only radiation but also other stressors such as toxic chemicals, preparing future disasters and supporting risk communication plans during recovery periods after disasters.
\end{abstract}

Keywords: risk communication / Fukushima nuclear accident / radiological protection / public understanding

\section{Introduction}

After the Fukushima nuclear accident in Japan, a number of practical activities related to public understanding (PU) of radiation risks were implemented inside and outside Fukushima prefecture. Although the large amount of information provided to people does not appear to have significantly improved PU of radiation risks, the quality of the information may have only accelerated the bifurcation of the public debate because of different ideas and opinions expressed by radiation experts via the news media (Science Council of Japan, 2014).

*Corresponding author: w-naito@aist.go.jp
The experience with PU of Genetically Modified Organisms (GMO) and Bovine Spongiform Encephalopathy (BSE) issues in UK has led to a shift from a "deficit model" style of communication to two-way communication between the public and scientific communities. In the immediate aftermath of the Fukushima nuclear accident, many radiation risk communication practices were a form of the "deficit model" communication style; as time passed, interactive or collaborative two-way styles of communication have appeared (Horikoshi et al., 2019). A number of interactive practical activities, including pragmatic researches related to PU of radiation risk, gradually emerged after the Fukushima nuclear accident. Innovative or unique forms of practical PU activities, such as the social networking service (SNS) and citizen science 
approaches, were also realized. Amidst growing anxiety about radiation risks in the wake of the accident, citizen scientists in Japan and abroad voluntarily collected information on radiation exposure and disseminated it to the public using SNS and other means, apart from official information sources (Brown et al., 2016). Citizen science is the practice of public participation and collaboration in scientific activities, such as the collection and analysis of empirical data. In recent years, the use of advanced information technology such as smartphones and GPS receivers has become more popular in collecting scientific data, especially in Europe and the United States. This is emblematic of the nature of PU activity in the era of information and communication technology (ICT). Coexpertise processes (Lochard et al., 2020) and communitybased approaches were also observed in affected areas of Fukushima. Many activities related to PU of radiation risks implemented in Japan are not well organized or documented in the literature, are sometimes documented only in Japanese, or are inconspicuous, making it impossible to refer to them even if they could contribute to important lessons regarding the PU of radiation risks in the future.

Horikoshi et al. (2019) examined approximately 500 pragmatic researches and activities related to radiation risk reduction and communication after the Fukushima accident using cross tabulation, text mining, and correspondence analysis. They evaluated the contributions of academia/experts and revealed that the main practical activities changed over time and that the activities differed by area. Murakami et al. (2017) reviewed medical professionals' risk communication activities in Fukushima Prefecture from the prefectural level to the individual level and found that the activities generally started with communication about radiation risks, mainly through group-based discussions, but gradually shifted to faceto-face communications to address comprehensive health risks to individuals and their well being. They observed that the purpose of these activities shifted from "promoting scientific understanding" to "supporting the decision-making of residents (Engagement)". Yamaguchi et al. (2018) reviewed risk communication guidelines and public health activities regarding radiation risk communication after the Fukushima disaster and concluded that successful practices are those that are helpful to the local community, supported by the local community, and observable in the local community, and that are supported by strategic approaches with a team organized by many experts in local areas, including local risk communicators.

Although the above-mentioned studies provided valuable insights into the activities related to PU of radiation risk, few studies have reviewed the practical PU activities of radiation risk implemented after the accident or attempted to make them knowledgeable or to discuss lessons learned in order to prepare for future nuclear accidents. Various approaches and strategies underlie the activities related to $\mathrm{PU}$ of radiation risks conducted after the accident, but these have not been well organized or made explicit thus far. In the current paper, we have organized the noteworthy activities related to PU of radiation risks implemented after the accident and discussed them mainly from the standpoint of communication strategies and approaches.

\section{Methods}

Examples of practical activities related to PU of radiation risk after the Fukushima accident were collected from the database (about 500 in total) created in Horikoshi et al. (2019), as well as from academic papers, government reports, websites, and books. The database from Horikoshi et al. (2019) covered practical activities reported between 2011 and March 2017. The first author first selected candidate examples for analysis, and then, based on discussions among co-authors, determined which examples to analyze. We selected those that featured either twoway communications among stakeholders or an innovative or unique means of communication. Each extracted example was systematically organized using an information organization sheet. Each example was analyzed and discussed from the perspective of communication approaches and strategies, and an attempt was made to identify characteristics and lessons learned that could be used as a reference for future activities to improve $\mathrm{PU}$ of radiation risk.

\section{Results}

\subsection{Overview of selected practical activities related to $\mathrm{PU}$ of radiation risk}

Examples of the practical activities covered in this analysis are shown in Table 1. Twenty-one examples were extracted and categorized into five categories: community-based approach (CBA; seven examples), researcher (including academic institution)-driven approach (RDA; two examples), dialogue and meeting (DM; two examples), citizen science approach (CSA; two examples), and innovative or unique communication strategies and approaches (IUCSA; eight examples). The characteristics of the examples in each category are described below. Although categorization is not a common practice, it is organized according to these categories for convenience. There are also examples of practical activities that include elements of more than one category.

In the present paper, CBA is defined as an approach in which affected communities are actively engaged in planning, monitoring, and evaluating the radiological situation of the local environment with the help of experts. In the selected examples, the community or local residents play a central role in understanding radiological situations in their local environments. The process of measuring and interpreting their own radiation levels in their environment by themselves, sometimes with the help of experts, is a hallmark of these examples. Although radioactivity measurement is a necessary component in the selected PU activities, the ultimate objectives of the activities addressed here were to determine whether it was possible to live safely, reclaim daily life, or start agricultural activities in affected areas, or whether further measures were needed. In addition, some activities introduced here have been recognized as good examples of a co-expertise process in the aftermath of the accident (Schneider et al., 2019; Lochard et al., 2020).

RDA is defined as an approach in which PU activities related to radiation risk are driven mainly by institutional and academic researchers. There are many examples of such 


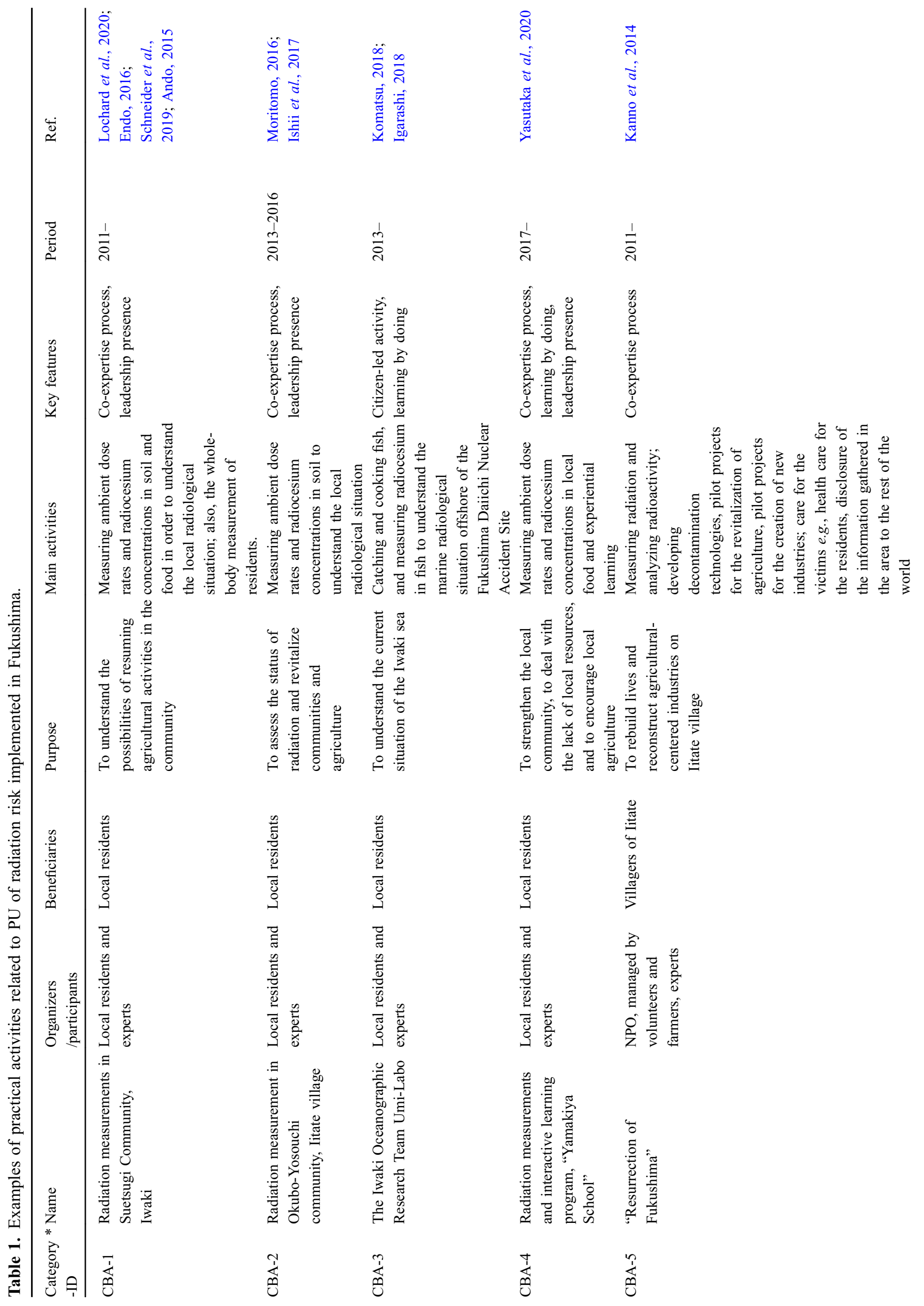




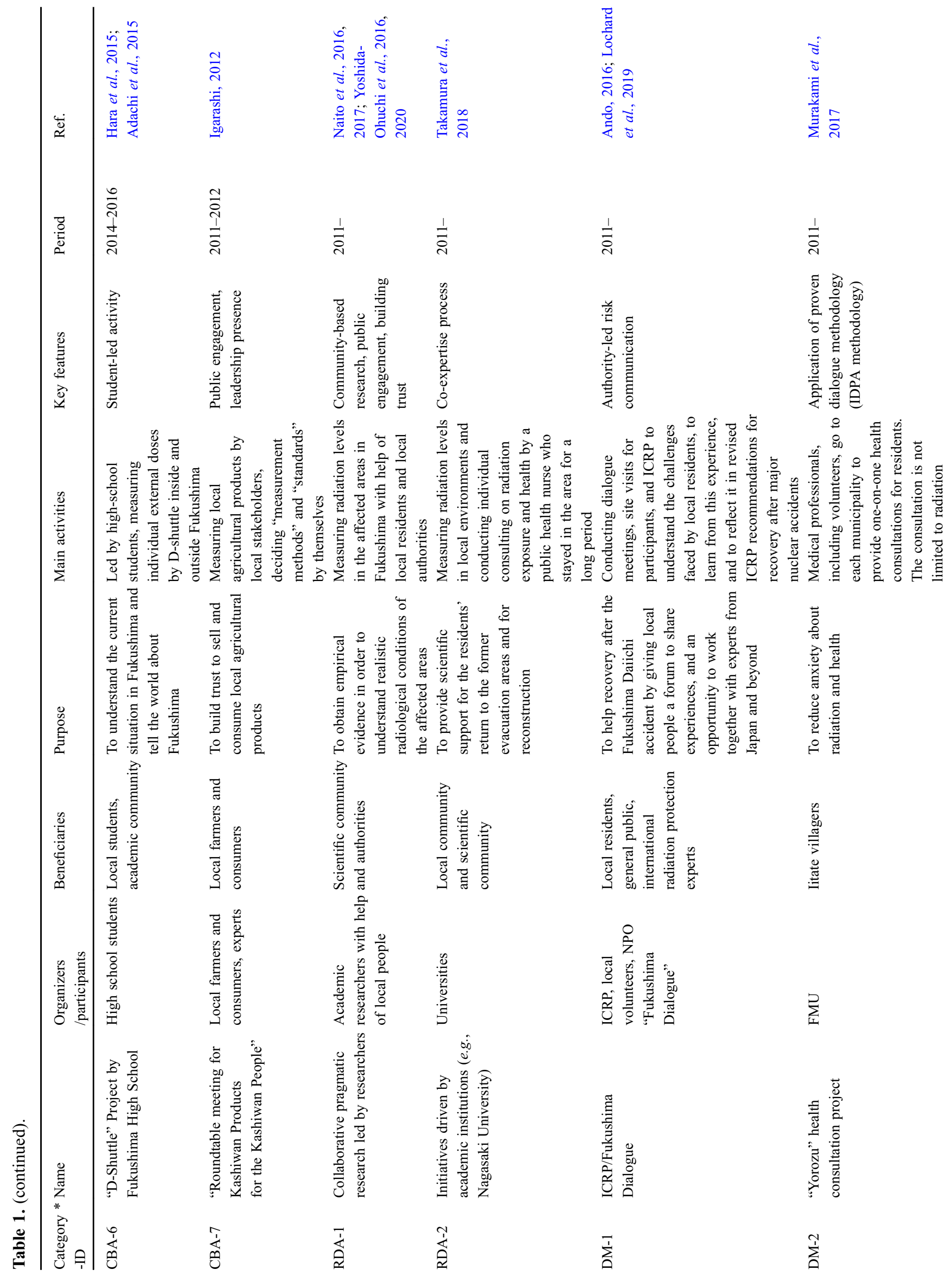




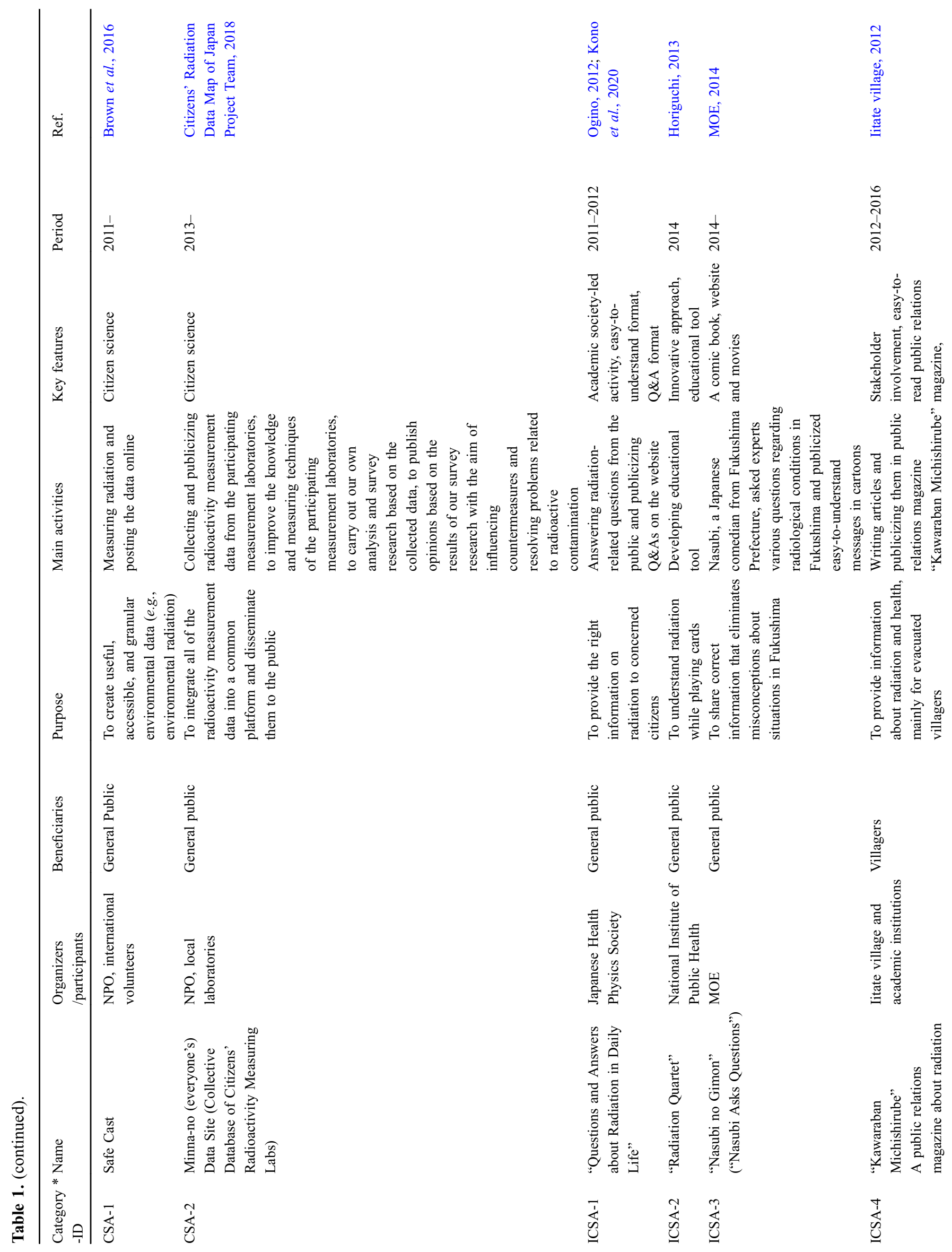




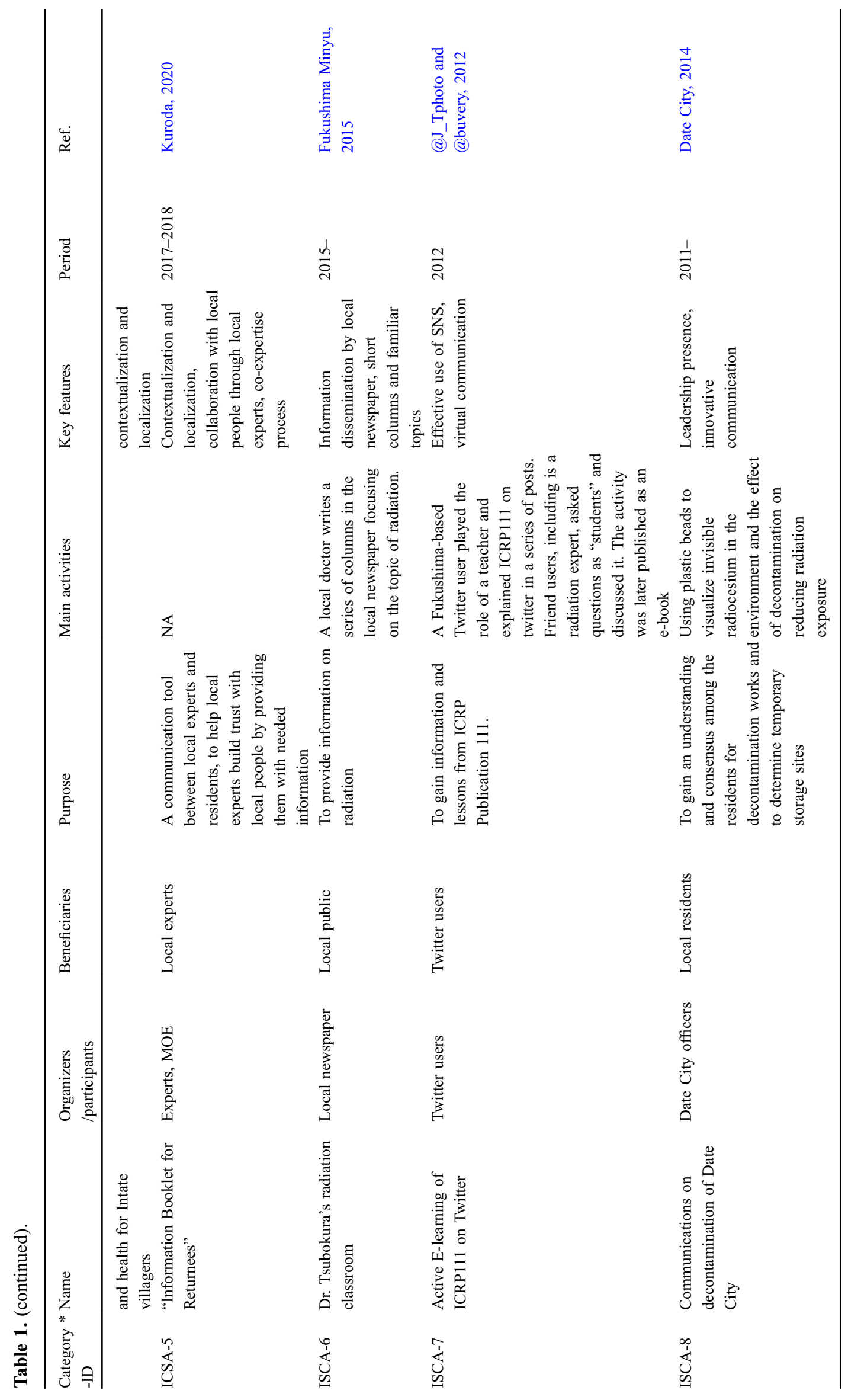


initiatives conducted by researchers in cooperation with residents, such as (Naito et al., 2016, 2017; Yoshida-Ohuchi et al., 2016, 2020). Researchers from outside Fukushima, with the cooperation of local residents, measured personal or air doses of radioactivity in living environments such as houses, and explained the results to the residents. Some universities set up satellite offices in the evacuation municipalities and conducted risk communication for residents. In the village of Kawauchi and the town of Tomioka, the Nagasaki University has been involved in communication with residents by stationing specialists in the satellite offices to build a sense of trust with them (Takamura et al., 2018). The cases selected here were distinctive in that not only were the results of individual measurements fed back to the participants or local community, but the results were summarized in academic papers and often utilized as reference materials in municipal and government decision-making processes.

Many dialogues and meetings were held to improve PU of radiation risk after the Fukushima accident, such as ICRP Dialogue/Fukushima Dialogue and the activities conducted by Fukushima Medical University. ICRP Dialogue/Fukushima Dialogue was a series of dialogue meetings involving national and international experts and local residents (Lochard et al., 2019). Flexible themes were discussed, depending on the time of year, and were used to build relationships and share awareness of issues. The application of a proven dialogue method, i.e. IDPA (Identification, Diagnosis, Prospective, Action proposals) method (EURANOS, 2009), contributed to the sharing of values, the promotion of mutual understanding, and an understanding of the diversity of "interpretations" among residents. In the Yorozu ("general") Health Consultation, one of many risk communication activities initiated by Fukushima Medical University, medical personnel visit municipalities to provide health consultations for local residents (Murakami et al., 2017). Hundreds of volunteer medical personnel from all over Japan participated as consultants. Consultations are held during events such as mass medical checkups in municipalities and are designed to reduce the avoidance of learning about radiation by creating a "face-to-face relationship" through one-on-one communication and not limiting the consultation to the subject of radiation. These examples are unique in that they are designed to be places where participants can share their experiences and thoughts not only about radiation, but also about their present and future life as well as their health concerns regarding living in the affected areas.

Citizen science refers to scientific activities performed by amateur scientists. Following the Fukushima accident, some citizen science groups endeavored to fill data gaps, as citizen group members in Japan measured radioactivity in the environment and communicated the results via the Internet (e.g., Brown et al., 2016; Citizens' Radiation Data Map of Japan Project Team, 2018). Citizen science activities addressed here played an important role in improving the PU of radiation by confirming the reliability of radioactivity measurements in the environment by the government and obtaining data in areas the government does not cover.

A number of innovative and unique initiatives were implemented to improve the PU of radiation risk following the Fukushima accident. These include preparing materials that respond to residents' actual concerns, using unique visual effects to explain the current radiological situation and decontamination, communication through games and online information-entertainment learning programs, and using social networking sites such as Twitter for active learning sessions.

\section{Discussion (lessons learned)}

As summarized above, during the recovery phase after the Fukushima accident, many unique and interesting PU initiatives and practices were implemented inside and outside Fukushima. We believe it is possible to identify several key lessons for improving PU practices for radiological risk and protection, especially in the recovery phase after a nuclear accident. In the following section, we discuss several important tips learned from those examples.

\subsection{Contextualization and localization of radiation risk}

Contextualizing radiation risks in the context of everyday life could help the public understand radiation risks. A number of risk communication efforts presented in this paper have been undertaken to convey the risks of radiation in the context of local life and needs. For example, communitybased monitoring programs working with scientists to measure and understand radiation in the living environment have been implemented elsewhere in the post-accident recovery (Dubreuil et al., 1999) . They include measuring internal and external radiation exposure as well as radiation levels in locally produced foods. Measuring radiation was necessary for local residents to understand the radiological conditions in their living environment as they sought to resume livelihoods and agricultural activities in the affected areas. "Information Booklet for Returnees", published by the Japanese Ministry of Education (MOE), was created for local counsellors to use as a communication tool to address the concerns and needs of returnees (Kuroda, 2020). Questions raised in the booklet were linked with residents' actual concerns of radiation risk in the context of their everyday life. The Yorozu ("general") health consultation project led by FMU started one-to-one health consultations with local residents; the program was run by local government public health nurses in 2012. They set up booths at public healthcheck venues, listened to people's concerns, responded individually to questions from the nurses, and provided educational material for them (Murakami et al., 2017). Such general health consultations could provide opportunities for local residents to share their local or individual concerns about radiation exposure and other health issues. Local public health nurses played an important role by listening and responding to various health concerns of those living in affected areas. Measurements by a whole-body counter (WBC), which measures internal radiation levels within the body, have been used to communicate risk between medical professionals and local residents in Fukushima (Hayano et al., 2014; Tsubokura et al., 2020). In Minamisoma Municipal General Hospital, a physician explained WBC results to each examinee and offered outpatient counseling to people with $>20 \mathrm{~Bq} / \mathrm{kg}$ of ${ }^{137} \mathrm{Cs}$ to discuss lifestyle choices, with a focus on food selection. Such consultations provide an opportunity 
for people to understand radiation exposure in their daily lives.

The experiences gained from PU activities after the Fukushima accident demonstrated that the contextualization and localization of radiation risks in the living environment can be effective at improving PU of radiation risks. A similar lesson was learned from Brian Wynne's study of Cumbrian sheep farmers, in which Wynne emphasized "knowledge in context" and illustrated the need for improved, two-way communication between scientists and the involved public during emergency situations (Wynne, 1989). Efforts to contextualize and localize radiation risk in various forms could provide valuable information with which to prepare and implement communication strategies and approaches that help the public understand radiation risk in living environments.

\subsection{Public involvement in radiological protection}

After the Fukushima accident, a variety of public-involved studies and monitoring programs were implemented to enhance people's understanding of radiological situations. Public-involved studies or monitoring programs can be broadly divided into two approach types: top-down and bottom-up. In the top-down approach, formal institutions such as research institutions, universities, and NPOs initiate public engagement and citizens collect radiation measurement data for them. On the other hand, the bottom-up approach involves public-initiated or public-driven practices, such as measuring ambient dose rates in the environment or concentrations of radioactive materials such as ${ }^{137} \mathrm{Cs}$ in food items. In the postaccident situation in Fukushima, both the top-down and bottom-up approaches certainly were taken and both played important roles in improving the PU of radiation risks. The involvement of Nagasaki University with Kawauchi village (Takamura et al., 2018), a community-based participatory radiation measurement in Yamakiya (Yasutaka et al., 2020), and individual dose measurements designed by institutional researchers (Naito et al., 2016) can be categorized as examples of the top-down approach. While there have been numerous top-down practices, including examples presented here, the selected examples are unique in that these activities are long lasting and have been adapted to changing circumstances. Although the primary purpose of top-down approaches led by formal institutions or institutional scientists was to obtain scientific knowledge in the affected areas, in some cases the monitoring activities evolved into activities that contributed to solving local problems through close interaction with local residents rather than just acquiring radiation data by researchers (Yasutaka et al., 2020). Community-based radiation measurement studies conducted by the present authors (Naito et al., 2017) in the affected areas in Fukushima met the needs of the local residents and helped them to understand the actual radiological situations in their living environment and their own radiation exposure. The dialogue with the local residents provided a good opportunity for the researchers to learn about residents' needs, and their local knowledge was very helpful in interpreting the measurement data. The proactive involvement of local residents is important in understanding and solving radiological risk problems, as the case of Fukushima confirmed. The measurement of radiation by residents can be a solution to a problem (e.g., relieving anxiety), but it can also be limited to the discovery of a problem (e.g., discovery of a high dose level or the generation of anxiety). Appropriate countermeasures cannot always be presented. When conducting research in collaboration with local residents, it is necessary to pay attention to the possibility that the research results may negatively impact both the local residents and society.

While the cases mentioned above were targeted to local residents in the affected areas of Fukushima, some activities involving the general public at the national and international levels were also noteworthy. Safecast and Minna-no-data-site ("Everyone's Data Site"), which are categorized as citizen science approaches, have been collaborating with the public on collecting and sharing radiation data. Their activities could provide insightful data that can promote $\mathrm{PU}$ of radiation exposure in the environment.

\subsection{PU of radiation risk in the era of ICT}

The growth of information and communication technologies has made social media popular for disseminating radiation risk messages by citizens, experts, and agencies. The Fukushima nuclear accident was the first large-scale nuclear disaster to occur in the era of ICT. After the accident, the results of radiation measurements and monitoring information from various entities were released on websites and via SNS. SafeCast and Minna-no-data-site are typical of those cases. A noteworthy initiative was an interactive study group that emerged in virtual space on SNS. The idea was that experts became the commentators and non-experts became the learners, reading and understanding ICRP 111. This twoway learning exchange became a publication (@J_Tphoto and (a) buvery, 2012). Due to the nature of Twitter, information is provided mainly in one direction, but a virtual community like this one attempted to search for two-way communication on the Internet. The process of scientific data acquisition and dissemination by the public or citizen scientists is expected to become more widespread. The impact on PU of science can be significant. On the other hand, there are various challenges, including ethical issues. Ethical issues related to citizen science should be addressed when projects start and throughout the course of scientific and communication activities.

\subsection{The presence of driving forces or mediators in PU activities}

For the PU activities implemented after the Fukushima nuclear accident, some individuals have been important driving forces or mediators, such as founders of NGOs, local farmers, local government officials, medical doctors, and scientists. Such personnel were clearly present in examples of CBA-1, CBA-2, CBA-4, and ISCA-8. Some experts have supported the residents' data collection, analysis, and interpretation, and acted as mediators between residents and authorities or residents and experts. Schoolteachers, public health nurses, and administrative ward chairpersons who worked in the affected areas before and after the accident, also have been instrumental in helping the affected residents. An administrative ward chairperson used their traditional 
community decision-making channel to initiate a communitybased radiation monitoring program. The presence of such personnel could help the public understand the radiological situation and their own exposure to radiation, and could help them regain confidence that there is a future for the affected areas in Fukushima. Honda et al. (2020), who conducted interviews with ten risk communicators after the accident, suggested that having professional and empirical knowledge founded on professional ethics is an important element that supports and facilitates risk communication works. The presence of driving forces or mediators who consistently conduct their work and activities based on professional ethics and expertise can also help to promote PU activities and build trust among stakeholders.

\subsection{Limitations and challenges}

A few limitations worth noting. First, this study does not cover all the PU activities implemented following the Fukushima nuclear accident. For example, there is limited discussion of practical activities for PU related to radiation risk that were implemented by medical professionals. Undocumented resident-driven activities are not covered. We may have eliminated valuable practical activities that include relevant information. However, we are confident that the examples selected, as well as the lessons learned from them, adequately covered the important aspect of PU of radiation risk, since the examples were selected by authors who have been involved in PU activities in Fukushima and discussion in the Task Group on PU activities after the accident in the Japan Health Physics Society. Another limitation is that the identification of good practices was a subjective process from the viewpoints of experts. Evaluations of PU activities from recipients' perspectives are missing. In order to objectively evaluate the effectiveness of practical activities related to PU of radiation risk, it is also necessary to evaluate what risk communication has been effective for residents and what has been helpful from the public's perspective.

One challenge is how to spread the good PU practices of one community to other communities. The purpose of a community-based approach led by local people was to solve problems in one's own community, and these efforts by themselves did not provide much in the way of support or development in other areas. The presence of enthusiastic personnel is an important element of good practice, but not all communities have such personnel. In their absence, it would be important to consider what mechanisms and systems need to be in place. Opportunities such as interregional exchange dialogues could contribute to aid and develop a form of PU that meets the needs of the area.

Another challenge is ensuring the sustainability of budgets and human resources for PU activities. The continuity of practical activities requires a budget. The continuity of such activities to obtain scientific evidence also depends on budgets and human resources. While some activities are sufficient for a successful PU as part of a transitory effort, they often require continuous monitoring. Continuous efforts are also important for building trust between residents and scientists.

\section{Conclusion}

In this study, we have reviewed the noteworthy activities to improve PU of radiation risks conducted after the Fukushima, Japan, nuclear accident in terms of communication strategies and approaches, and discussed lessons learned and challenges remaining. During the post-accident recovery phase, a wide range of practical activities related to $\mathrm{PU}$ of radiation risks were conducted inside and outside Fukushima. We draw several key lessons and challenges for improving PU practices for radiological risk and protection, especially in the recovery phase of a post-nuclear accident. As seen in the various examples, efforts to contextualize and localize radiation risk in various forms were observed during postaccident recovery, and these efforts were confirmed, through actual experiences, to be an important component of effective PU activities of radiation risks. There were several noteworthy examples of community-based or citizen science approaches to radiation risk PU practices implemented in Japan. Experiences with such approaches, such as residents measuring radiation levels, have been proven to contribute to PU of radioactive situations, but some challenges, such as ethical issues and the handling of uncertainty, should be noted. As the Fukushima nuclear accident was the first largescale nuclear disaster to occur in the era of ICT, a number of citizens, experts, and agencies have made social media a popular way to disseminate radiation risk messages to the public and demonstrated that social media can play important roles in providing radiological risk information. Moreover, the presence of driving forces or mediators who consistently conduct their work and activities based on professional ethics and expertise can also contribute to PU activities and help build trust among stakeholders.

Fitzpatrick-Lewis et al. (2010) suggested that risk communication strategies incorporating the needs of local residents with a multifaceted delivery method are most effective at reaching a target audience. While the PU of science is important, it is also important for scientists to understand the public (Fujigaki et al., 2008). Many of the activities brought about by experts after the Fukushima accident were aimed primarily at improving the PU of radiation risk, that is, at providing knowledge to the public. On the other hand, experts themselves considered their interactions with the population and its ethical norms, leading them to turn to activities such as public engagement, which are characterized by activities to support the decision-making of the population (Murakami et al., 2017). The body of knowledge and lessons learned from practical activities introduced and discussed in this study can be useful in enhancing PU of not only radiation but also other disaster situations such as chemical accidents. They can also help us prepare for future disasters and support risk communication plans during recovery periods.

\section{Acknowledgments}

This work was mainly conducted in the JHPS's "Task Group on Public Understanding after the Fukushima Daiichi Nuclear Power Plant Accident" in the FY2018-2019. This 
work was partially supported by JSPS KAKENHI Grant Number 16KK0019.

\section{References}

@ J Tphoto and @ buvery. 2012. Introduction to ICRP Publ.111. http://birdtaka.com/download/intro-icrp111/Intro_ICRP111_sin gle_1_2_1.pdf (retrieved 19, Nov., 2020, Japanese).

Adachi $\overline{\mathrm{N}}$, et al. 2015. Measurement and comparison of individual external doses of high-school students living in Japan, France, Poland and Belarus - the 'D-shuttle' project. J. Radiol. Prot. 36 (1): 49-66.

Ando R. 2015. Measuring, discussing, and living together: lessons from 4 years in Suetsugi. Ann. ICRP 45(1 Suppl.): 75-83.

Ando R. 2016. Ethos in Fukushima and the ICRP dialogue seminars. Ann. ICRP 45(2_Suppl.): 135-140.

Brown A, et al. 2016. Safecast: successful citizen-science for radiation measurement and communication after Fukushima. $J$. Radiol. Prot. 36(2): S82-S101.

Citizens' Radiation Data Map of Japan Project Team. 2018. Citizens' radiation data map of Japan. Minna-no Data Site (Japanese).

Date City. 2014. Date City report since 2011.3.11. Date City (Japanese).

Dubreuil GH, et al. 1999. Chernobyl post-accident management: the ETHOS project. Health Phys. 77(4): 361-372.

Endo S. 2016. A farmhouse son-in-law and radiation. Ann. ICRP 45 (2_Suppl.): 71-77.

EURANOS. 2009. Sustainable rehabilitation of living conditions in contaminated territories after a nuclear accident or a radiological event - Revised framework for the elaboration of post-accident rehabilitation preparedness strategies. Deliverable Report for the EURANOS project. European Commission.

Fitzpatrick-Lewis D, et al. 2010. Communication about environmental health risks: a systematic review. Environ Health Glob Access Sci Source 9: 67-67.

Fujigaki Y, et al. 2008. Theoretical perspective for science communication (Japanese).

Fukushima Minyu. 2015. Dr. Tsubokura's Radiation Classroom. https://www.minyu-net.com/kenkou/housyasen/ (Retrieved 19, Nov., 2020, Japanese).

Hara T, et al. 2015. Radiation exposure level in senior high school students in Fukushima Prefecture in comparison with those in other prefectures. J. Phys. Educ. Soc. Jpn. 63(2): 87-91 (Japanese).

Hayano R, et al. 2014. BABYSCAN: a whole body counter for small children in Fukushima. J. Radiol. Prot. 34(3): 645-653.

Honda $\mathrm{K}$, et al. 2020. The structuralization of risk communication work and objectives in the aftermath of the Fukushima nuclear disaster. Int. J. Disaster Risk Reduct. 50: 101899.

Horiguchi I. 2013. Research on risk communication after the Fukushima nuclear power plant accident. J. Natl. Inst. Public Health 62(2): 150-156 (Japanese).

Horikoshi H, et al. 2019. Analysis on researchers' practical activities for radiation risk reduction and risk communication after the Fukushima Nuclear Power Plant accident. Jpn. J. Risk Anal. 29(2): 103-110.

Igarashi Y. 2012. The shape of "peace of mind" decided by everyone. Akishobo (Japanese).

Igarashi Y. 2018. The nuclear disaster and food - market, communication and discrimination. Chuokoron-Shinsha, Inc. (Japanese).

Iitate village. 2012. Kawaraban Michishirube. https://www.vill.iitate. fukushima.jp/life/5/19/75/ (Retrieved 19, Nov., 2020, Japanese).
Ishii $\mathrm{H}$, et al. 2017. Radiation measurement and experimental cultivation conducted by cooperation between residents and universities / research institutes. J. Cent. Reg. Aff. Fukushima Univ. 29(1): 8570-8580 (Japanese).

Kanno M, et al. 2014. Decontamination in collaboration with Iitate Village residents and research institutions. Trends Sci. 19(7): 3639 (Japanese).

Komatsu R. 2018. New revivalism. Genron Co., Ltd. (Japanese).

Kono T, et al. 2020. Analysis of the activities of the website "Question and answer about radiation in daily life" after the accident at the Fukushima Daiichi Nuclear Power Station and some lessons learned from it-To pass on this experience to the future. Jpn. J. Health Phys. 55(4) (Japanese).

Kuroda Y. 2020. The making of "Information booklet for returnees". Building trust through collaboration with local communities. Radioprotection 55(4): 309-315. https://doi.org/10.1051/radiopro/ 2020081.

Lochard J, et al. 2020. The post-nuclear accident co-expertise experience of the Suetsugi community in Fukushima Prefecture. Radioprotection 55(3): 225-235.

Lochard J, et al. 2019. An overview of the dialogue meetings initiated by ICRP in Japan after the Fukushima accident. Radioprotection 54(2): 87-101.

MOE. 2014. Nasubi no Gimon. http://josen.env.go.jp/nasubinogi mon/ (Retrieved 19, Nov., 2020, Japanese).

Moritomo Y. 2016. Continuation of farming and regional regeneration. J. Rural Plan. 34(4): 423-427 (Japanese).

Murakami M, et al. 2017. Communicating with residents about risks following the Fukushima Nuclear Accident. Asia Pac. J. Public Health 29(2_Suppl.): 74S-89S.

Naito W, et al. 2016. Relationship between individual external doses, ambient dose rates and individuals' activity-patterns in affected areas in Fukushima following the Fukushima Daiichi Nuclear Power Plant Accident. PLoS One 11(8): e0158879.

Naito W, et al. 2017. Measuring and assessing individual external doses during the rehabilitation phase in Iitate village after the Fukushima Daiichi nuclear power plant accident. J. Radiol. Prot. 37(3): 606-622.

Ogino H. 2012. Reflection on risk communication in 9 months after Fukushima Nuclear Accident. Jpn J. Health Phys. 47(1): 37-43 (Japanese).

Science Council of Japan. 2014. Committee of clinical medicine Radiological protection and risk management sectional committee: enhancement of radiological health risk science education including compulsory in medical education (Japanese).

Schneider T, et al. 2019. The role of radiological protection experts in stakeholder involvement in the recovery phase of post-nuclear accident situations: some lessons from the Fukushima-Daïchi NPP accident. Radioprotection 54(4): 259-270.

Takamura N, et al. 2018. Recovery from nuclear disaster in Fukushima: collaboration model. Radiat. Prot. Dosimetry 182(1): 49-52.

Tsubokura M, et al. 2020. Usefulness of the whole-body counter for infants and small children (BABYSCAN) as a risk communication tool after the Fukushima Daiichi nuclear power plant incident. Proc. Jpn. Acad. Ser. B 96(2): 70-78.

Wynne B. 1989. Sheepfarming after Chernobyl: a case study in communicating scientific information. Environment (U. S.) 31: 2.

Yamaguchi I, et al. 2018. Lessons learned from radiation risk communication activities regarding the Fukushima nuclear accident. J. Natl. Inst. Public Health 67(1): 93-102.

Yasutaka T, et al. 2020. Dialogue, radiation measurements and other collaborative practices by experts and residents in the former evacuation areas of Fukushima: a case study in Yamakiya District, Kawamata Town. Radioprotection 55(3): 215-224. 
Yoshida-Ohuchi $\mathrm{H}$, et al. 2016. Indoor radiocaesium contamination in residential houses within evacuation areas after the Fukushima nuclear accident. Sci. Rep. 6: 26412.
Yoshida-Ohuchi H, Shinohara N. 2020. Estimated internal exposure doses due to indoor radiocaesium contamination in residential houses after the Fukushima nuclear accident. Sci. Rep. 10(1): 17212.

Cite this article as: Naito W, Uesaka M, Kuroda Y, Kono T, Sakoda A, Yoshida H. 2020. Examples of practical activities related to public understanding of radiation risk following the Fukushima nuclear accident. Radioprotection 55(4): 297-307 